\title{
Vom Radioprogramm für die Leipziger Messe zur „Radio-DDR-Messewelle“ Informationen, Service, Unterhaltung und Werbung in unterschiedlicher Ausrichtung
}

\author{
von \\ CHRISTIAN KÖNNE
}

Immer informiert sein - die Radio DDR-Messewelle hören!

Слушайте Радио ГДР на волне ярмарки - и вы будете всегда в курсе!

Keep in Tune - Listen to Radio DDR-Messewelle

Pour être toujours bien informé, écoutez „Messewelle“

Die Leipziger Messe war eines der internationalen Großereignisse der DDR. Sie bot damit die Gelegenheit, die politische DDR medial ins rechte Licht zu rücken. Die Messe-Sondersendungen waren für die SED wichtige Beiträge in den ostdeutschen Programmen. Dementsprechend wurde die Wahrnehmung jeder der zweimal jährlich stattfindenden Messe durch eine Komiteevorlage des Staatlichen Rundfunkkomitees beim Ministerrat der DDR (SRK) festgesetzt. Die medial vermittelte Kommunikation anlässlich der Leipziger Messe diente dabei angeblich nicht nur dem Handel, sondern auch der Verständigung der Völker. Gleichzeitig war die Messe eine Leistungsschau der Wirtschaft unserer Arbeiter- und Banernmacht. ${ }^{1}$ Die Übertragungen aus Leipzig waren also Angebote für einen wirtschaftlichen und politischen Dialog sowie für das Ausland ein Fenster zur DDR. ${ }^{2}$ Das kam nicht von ungefähr. Aufbau des Sozialismus durch wirtschaftlichen Austausch und zunehmende Anerkennung der DDR durch Partnerschaften mit anderen Staaten - auch im Handel - waren die Grobziele, die es bis in die 1970er-Jahre $\mathrm{zu}$ verfolgen galt. Es gab ein Radioangebot der Zentralsender und auch eines des

1 Deutsches Rundfunkarchiv Potsdam-Babelsberg, Schriftgut Hörfunk (im Folgenden: DRA), Komiteevorlage des Staatlichen Rundfunkkomitees beim Ministerrat der DDR (im Folgenden: KV) 18/55, 23. August 1955, Politische Argumentation für die Herbstmesse 1955.

2 Zur Kommunikation mit der Bundesrepublik wurde der Regionalsender auch bei der Begegnung der Fußballmannschaften 1. FC Kaiserslautern gegen SC Wismut Karl-MarxStadt in Leipzig 1956 genutzt. Vgl. Christian KönNe, Für Einheit und Anerkennung. Die Fußballspiele des 1. FC Kaiserslautern gegen SC Wismut Karl-Marx-Stadt (Chemnitz) 1956/57, in: Kaiserslauterer Jahrbuch für pfälzische Geschichte und Volkskunde 10/11 (2012), S. 71-92, hier S. 79. 
Regionalsenders Leipzig, die an der Entwicklung der späteren „Messewelle“ einen Anteil hatten.

\author{
I. Vorgeschichte der „Messewelle“ Teil 1 - \\ Radioprogramm zur Leipziger Messe in den Zentralprogrammen
}

\title{
Die Propagierung des Sozialismus
}

Verschiedene Sendungen anlässlich der Messe wurden entsprechend ihres politischen Stellenwerts über die drei zentralen Programme Radio DDR, Berliner Rundfunk und Deutschlandsender ausgestrahlt. Radio DDR informierte beispielsweise täglich umfassend und aktuell über kommerzielle, kulturelle und politische Höhepunkte auf dem Welthandelsplatz Leipzig. Die Sonderredaktion produzierte montags bis freitags zwischen 16.00 und 17.00 Uhr im I. Programm die „Messe-Revue“. Auf Radio DDR II lief täglich von 18.15 bis $18.30 \mathrm{Uhr}$ das „Messe-Interview“. Daneben gab es auf Radio DDR Berichte und Informationen [...] in allen aktuellen Sendungen. ${ }^{3}$ Für Radio DDR war „Leipzig - Treffpunkt der Welt“, wie es ein programmatischer Titel nannte. ${ }^{4}$ Um sich auf diesem adäquat zu unterhalten, war der Hörfunk auch für die öffentlichen Veranstaltungen im Kulturprogramm anlässlich der Messe zuständig. Die Planungen dafür lagen in den Händen der zentralen Musikabteilung in Berlin. ${ }^{5}$ Hier stellte die DDR ihr bestes Personal und ihre musikalischen Highlights dem internationalen Publikum vor. ${ }^{6}$ So wurde beispielsweise die Jubiläumssendung anlässlich von 15 Jahren „Schlagerrevue“ mit Heinz Quermann zur Eröffnung einer Herbstmesse in Leipzig präsentiert. ${ }^{7}$

Doch während sich der Sozialismus in Leipzig international feierte, war es um die finanzielle Situation der DDR zu Beginn der 1960er-Jahre äußerst schlecht bestellt. ${ }^{8}$ Es war Zeit, auch im Hörfunk von der Propagierung zur Finanzierung des Sozialismus überzugehen. Das tat man mit verschiedenen Sendereihen für die

3 FF Funk und Fernsehen der DDR 35/1966, S. 31.

4 DRA, KV 19/64, 28. Januar 1964, Frühjahrsmesse 1964.

5 Wolfgang TiETZ, Erinnerungen [Typoskript]. Bestand beim Verfasser. Ich bedanke mich bei Herrn Tietz für die Informationen!

6 Christian KÖNNE, „Schlagerrevue“ und „Schlager-ABC“. Hörfunksendungen zwischen Partei und Publikum, in: Sascha Trültzsch/Thomas Wilke (Hg.), Heißer Sommer Coole Beats. Zur populären Musik und ihren medialen Repräsentationen in der DDR, Frankfurt am Main u. a. 2010, S. 139-157. Vgl. zu den Messemusiksendungen im DFF auch: Uwe BREITENBORN, Wie lachte der Bär? Systematik, Funktionalität und thematische Segmentierung von unterhaltenden nonfiktionalen Programmformen im Deutschen Fernsehfunk bis 1969, Berlin 2003, S. 152-155.

7 DRA, Pressearchiv, Neue Zeit, 24. August 1968.

8 André Steiner, Von Plan zu Plan. Eine Wirtschaftsgeschichte der DDR, Berlin 2007, S. $139-180$. 
ostdeutsche Bevölkerung. ${ }^{9}$ Anlässlich der Messe erfolgte zu dieser Zeit auch eine intensivere Zuwendung zu den internationalen Handelspartnern.

\section{Veränderungen: Finanzierung des Sozialismus durch Radio DDR}

Seit Beginn der 1960er-Jahre hatte der Hörfunk für die Betreuung ausländischer Messegäste einen separaten Etat. Er forderte nun spezielle Räume mit entsprechender Ausstattung und Verpflegung für die Gäste anlässlich der Planung der Messen an. ${ }^{10}$ Man kümmerte sich um die westlichen Handelspartner - die Abgrenzung musste der Kooperation zur Finanzierung des Sozialismus weichen.

In den Unterlagen zum Jahr 1964 findet sich für die Planungen des Messe-Programms der Hinweis auf eine bemerkenswerte Programmveränderung, die eine Abwendung von der Propaganda und eine konkrete Hinwendung zu den Finanzen bedeutete. Als in der DDR das „Neue Ökonomische System der Planung und Leitung“ (NÖS) eingeführt wurde, übertrug der ostdeutsche Hörfunk nun auch Werbung. ${ }^{11}$ In Musik eingebettet, wurde in der Messe-Sondersendung auf Radio DDR zwischen 17.00 und 18.00 Uhr Messe-Reklame betrieben, d. h., [...] Erzengnisse vorgestellt. ${ }^{12}$ Die Reklame im Radio war zwar so ungewöhnlich, dass sie erläutert werden musste, doch der Zeitpunkt für die Hereinnahme der Werbung war offenbar günstig, denn bereits einen Monat später informierte der Hörfunk die Agitationskommission beim ZK der SED darüber, dass kapitalistische Firmen, die in Leipzig ausstellen, an Werbesendungen in unserem Rundfunk interessiert sind. War die Hörfunkwerbung anlässlich der Frühjahrsmesse 1964 eine Art Testballon im Hinblick auf die Reaktion der Aussteller? Der Hörfunk hatte nach dem Angebot der westlichen Firmen jedenfalls flugs die Überlegung geboren, dass mit den Geldern aus der zukünftigen Werbung eine Finanzierungsquelle für die Erweiterung des Auslandskorrespondentennetzes erschlossen werden könnte. ${ }^{13}$ Gleichzeitig dachte man darüber nach, ob wir mit diesen zu erwartenden Devisen möglicherweise moderne rundfunktechnische Einrichtungen kaufen, die [...] dringend notwendig sind. ${ }^{14} \mathrm{Im}$ Werbefunk stimmten die Wünsche der Aussteller mit denen des DDR-Hörfunks jedenfalls überein. Entsprechend der finanziellen

9 Christian KÖNNe, Neue Formen der Propaganda? Wirtschaftsagitation im Funk, in: Klaus Arnold/Christoph Classen (Hg.), Zwischen Pop und Propaganda. Radio in der DDR, Berlin 2004, S. 133-149.

10 DRA, KV 91/62, 26. Juli 1962, Herbstmesse 1962. Diese Anforderungen finden sich auch in den nachfolgenden Komiteevorlagen zur Vorbereitung der Messe.

11 Steiner, Von Plan zu Plan (wie Anm. 8), S. 151.

12 DRA, KV 68/64, 13. Juli 1964, Herbstmesse 1964.

13 Bundesarchiv Berlin-Lichterfelde (im Folgenden: BArch), Stiftung Archiv der Parteien und Massenorganisationen der DDR (im Folgenden: SAPMO), DY 30 IV A 2902 107, 17. August 1964, Abteilung Agitation, Protokoll über die gemeinsame Beratung der Agitationskommission mit dem SRK über die Perspektive des Deutschen Demokratischen Rundfunks und das Verhältnis zwischen Rundfunk und Fernsehfunk am 13. August 1964.

14 BArch, DR 6-607, 22. Oktober 1964, Brief an das Zentralkomitee der SED von Reginald Grimmer. 
Bedürfnisse des Funks wurde von diesem das Projekt eines Werbefunks während der Leipziger Frühjabrsmesse 1965 forciert. Doch durfte der Funk nicht allein der Befürworter und Nutznießer seiner politisch heiklen Neuerung sein. Deshalb gab er hierzu im Oktober 1964 folgende Einschätzungen wieder, die das kollektive Interesse am Werbefunk bekundeten: ${ }^{15}$

1. Es besteht besonders auch bei kapitalistischen Ausstellern dafür reges Interesse.

2. Das Leipziger Messeamt steht unserem Vorbaben positiv gegenüber und würde es entsprechend unterstützen.

3. Das Ministerium für Außenhandel und Innerdeutschen Handel sowie die Kammer für Außenhandel haben ebenfalls unsere Idee begrüßt und ibre Unterstützung zugesagt. Und

4. [es] ist auch das Ministerium der Finanzen an diesem Projekt interessiert.

Das delikate Projekt hatte also breite Unterstützung. Der „Werbefunk“ mochte politisch zwar erstaunlich sein, doch er bot die Möglichkeit, „harte Devisen zu bekommen". ${ }^{16}$ Und der ökonomische Zustand erforderte offenbar die mediale Verwendung aller wirtschaftlich verwertbaren Inhalte, um den Staat wirtschaftlich zu stabilisieren. Vom westlichen Ausland bezahlte Werbung war eine von ihnen. $\mathrm{Da}$ die Finanzen nicht allein als Argument für die Hereinnahme von ,WestWerbung' dienen durften, hatte diese neben der finanziellen angeblich auch eine politische Seite. Hier hielt der DDR-Hörfunk klar Kurs, denn der „Werbefunk“ sollte die Internationalität der Leipziger Messe und damit der Deutschen Demokratischen Republik [...] unterstreichen. Um dies zu erreichen, wurde jedoch bemerkenswerter Weise nicht über die eigenen internationalen - wirtschaftlichen - Erfolge berichtet und dementsprechend die sozialistischen Produkte beworben. Um die internationale Bedeutung des nicht anerkannten zweiten deutschen Staates deutlich zu machen, sollten im „Werbefunk“ Länder wie England, Frankreich, Italien und Belgien ins Zentrum gerückt werden, die mit ibren Investitionsgütern (Chemieanlagen z. B.) für unsere Volkswirtschaft von besonderem Interesse sind. Der Werbefunk wird also nicht Reklame für den Kapitalismus machen oder westliche Erzengnisse der Konsumgüterindustrie anpreisen. Der Werbefunk wird eine politische Aufgabe zu erfüllen haben, und so sind auch verantwortliche politische Redakteure unserer Sender für die Verwirklichung dieses Vorhabens vorgesehen. ${ }^{17}$ Der „Werbefunk“ war also ein politisch motiviertes Dankeschön an die westeuropäischen Staaten, die mit der DDR wirtschaftlich kooperieren wollten,

15 Ebd.

16 BArch, DR 6-607, 22. Oktober 1964, Brief an das Zentralkomitee der SED von Reginald Grimmer. Zitiert nach: CHRISTIAN KÖNNE, Ferienwelle und Messewelle und ihre Funktionen im Sozialismus, in: Stefan Zahlmann (Hg.), Wie im Westen, nur anders. Medien in der DDR, Berlin 2010, S. 326-342, hier S. 334.

17 BArch, DR 6-607, 22. August 1964, Brief an das Zentralkomitee der SED von Reginald Grimmer. 
zeigte er doch, dass die DDR - auch bei westlichen Staaten - bereits ein anerkannter (Handels-)Partner war. Hier wurde die Abgrenzung zur Bundesrepublik markiert, die im Kontext der sogenannten Störfreimachung der DDR-Wirtschaft gegenüber der Bundesrepublik zu sehen ist. Gleichzeitig waren dies vor der internationalen staatlichen Anerkennung politisch zentrale Aussagen in Bezug auf die Eigenstaatlichkeit der DDR.

Zur Kontaktierung im Vorfeld der Messe hatte die DDR beispielsweise in London eine „Leipzig Fair Agency“ und auch in Paris konnte man sich für die Leipziger Messe anmelden, denn dort gab es ebenfalls ein Büro, das den Interessenten an der Messe eine Kontaktmöglichkeit bot. Für Aussteller und Besucher wurde die 800-Jahrfeier der Leipziger Messe im Frühjahr 1965 seit Dezember 1964 auch in der westlichen Presse beworben. ${ }^{18}$

Doch blieben die Finanzen und die wirtschaftliche Kooperation unverändert die kurzfristig wirksamsten Effekte. Diese waren notwendig, denn die UdSSR hatte der DDR zu dieser Zeit ihre Lieferungen gekürzt, sodass Getreide oder Stahl im westlichen Ausland beschafft und chemische Produkte dorthin abgesetzt werden mussten. ${ }^{19}$

Die Idee, ausländische Werbung im Programm zuzulassen, war nicht im ostdeutschen Hörfunk entstanden. Das sozialistische Ausland war der Vorreiter, den man überflügeln wollte. Denn im Gegensatz zu polnischen und tschechoslowakischen Rundfunkstationen, die seit geraumer Zeit zu den Messen in Poznań und Brno Werbesendungen machen und dabei unserer Meinung nach zu unkommerziell verfabren, haben wir einen Preis kalkuliert, der beträchtliche finanzielle Einnabmen garantiert. Wir halten einen Preis von 200 US-Dollar bzw. 180 Rubel bzw. 836 DM-West für angebracht. ${ }^{20}$

Da die Situation in der DDR drängend war, setzte man die Ideen zügig um. ${ }^{21}$ Bereits zur Frühjahrsmesse 1965, also etwa vier Monate später, wurde dann ein internationaler Werbefunk übertragen, der die Werbung der ausländischen Kunden zu den genannten Preisen pro Minute übertrug. ${ }^{22}$

Die „Internationale Werbesendung“ kam dann im März 1965 im Programm von Radio DDR I und wurde dort unter der Ankündigung Nowy! New! Neuf in

18 The Times, Late London Air Edition, 1. Dezember 1964, S. 12; ebd., 15. Januar 1965, S. 21; Le Monde, 23. Dezember 1964, S. 22; ebd., 26. Januar 1965, S. 16; ebd., 7./8. Februar 1965, S. 7. In den Annoncen findet sich kein spezieller Hinweis eines Angebots für RadioWerbung.

19 STEINer, Von Plan zu Plan (wie Anm. 8), S. 155.

20 BArch, DR 6-607, 22. Oktober 1964, Brief an das Zentralkomitee der SED von Reginald Grimmer.

21 Im Frühjahr 1964 findet sich auf keinem DDR Sender eine explizite Werbesendung. Im Programm Radio DDR I lief als erste Sendung des Tages zwischen 11.00 und 11.30 Uhr und dann noch einmal von 19.00 bis 19.30 Uhr die Sendung „Leipzig - Treffpunkt der Welt“. Vgl. FF Funk und Fernsehen der DDR 10/1964, S. 24. Im Herbst liefen dann die „Radiotips am Morgen“ in der Zeit zwischen 7.33 und 7.57 Uhr, ohne dass ein Hinweis möglich ist, welche Inhalte hier genau übertragen wurden. Vgl. ebd. 37/1965, S. 22.

22 KÖNNE, Ferienwelle und Messewelle (wie Anm. 16), S. 334. 
der Zeit von 7.33 bis 8.00 Uhr übertragen, was während der gesamten Messedauer 189 Minuten Sendezeit entsprach. Um auch außerhalb des Radios auf sich aufmerksam zu machen, hatte der Hörfunk in der Programmzeitschrift „FF Funk und Fernsehen der DDR“ eine Annonce für seinen Werbefunk geschaltet:

Radio-DDR-Messefunk

Your product the GDR home market - Your commercial on GDR home Service!

Publicité à Radio DDR: fonds bien place, gage de success!

Publicidad con Radio DDR: sinónimo de éxite commerciáles!

Mit Radio DDR werben - beißt mit Erfolg und Nutzen werben!

Sie allein wissen es, wieviel Ihnen eine Minute bedentet, wir nicht. Aber wissen Sie, wie Sie Ihre Zeit auf der Leipziger Messe am besten nutzen können? Das wissen wir. Wir bieten Ihnen für Ihre Werbung unser Können und unser Wissen. Wir senden unsere Programme über 33 Frequenzen, im Mittel- und Ultrakurzwellenbereich. Auf Ihren Wunsch senden wir vom Sonntag, dem 28. Februar, bis Dienstag, dem 9. März, in Englisch, Französisch, Russisch oder Deutsch Ibre Werbesprüche. Schreiben Sie uns, oder besuchen sie uns, oder rufen Sie uns an, wieviel Zeit wir Ihnen reservieren sollen. Melden Sie sich bald, denn Zeit ist knapp, auch bei uns. Auch bei Ibnen müssen Sie die Zeit bezablen. Genau genommen 836,MDN die Minute oder 13,90 MDN die Sekunde.

Zweimal täglich: 7.30 bis $8.00 \mathrm{Ubr}$ und 18.00 bis $18.30 \mathrm{Uhr} .23$

Hier war nicht mehr die Rede davon, dass der Westen seine Produkte nicht bewerben dürfe, wie dies die internen Dokumente zuvor strategischer Weise behaupteten. Nun waren auch die Werbesprüche erlaubt, die zuvor angeblich nicht zugelassen werden sollten. Die finanziellen $Z$ wänge setzten den politischen Zielen den Rahmen. Dass die Ankündigungen nicht auf Russisch zu finden sind, wirft ein Licht auf das Verhältnis der DDR zur UdSSR zu dieser Zeit.

Der „Werbefunk“ übererfüllte die mit ihm verbundenen Planungen - das Interesse des Auslands war erfreulich groß. Schon vor der ersten "Werbesendung“ hielt man daher intern fest: Achtung: Aufgrund der Nachfrage ausländischer Interessenten muss bereits mit einem früheren Beginn der Werbesendungen als dem 23.2.65 gerechnet werden. Wabrscheinlich ab 15. Februar 1965.24 Mit dieser Ausdehnung wäre die Sendezeit des „Werbefunks“ um ein Drittel gesteigert worden, da die Übertragungen anlässlich der Messe zwei Wochen dauerten, hier jedoch eine weitere Woche hinzukommt. Das Interesse blieb anhaltend groß und die Sendezeiten des „Werbefunks“ auf Radio DDR I wurden in der Folge ausgebaut. 1966 lief er an den Wochentagen von 7.30 bis $8.30 \mathrm{Uhr}$ (mit einer 5-Minuten Pause

23 FF Funk und Fernsehen der DDR 10/1965, S. 22. In „The Times“ und „Le Monde“ finden sich parallel geschaltete Annoncen für den Werbefunk zwischen Oktober 1964 und März 1965 nicht. Allein die oben genannten allgemeinen Annoncen für die Leipziger Messe sind dort 1964/65 zu finden.

24 DRA, KV 119/64, 6. Dezember 1964, Frühjahrsmesse 1965. 
für die Nachrichten um 8.00 Uhr) sowie von 18.30 bis 18.55 Uhr. Samstags war er von 7.30 bis 8.00 und von 8.05 bis 8.30 Uhr zu hören, was einer Zunahme der Sendezeit binnen Jahresfrist um $269 \%$ entsprach. 25

Die Bedeutung, die der West-Werbung in der DDR eingeräumt wurde, zeigt sich auch in anderer Hinsicht. Für den "Werbefunk" wurde neben der Sonderredaktion zur Leipziger Messe, die seit jeher die übrigen Bereiche des Programms abdeckte, nun zusätzlich eine weitere Sonderredaktion eingerichtet. Der „Werbefunk" wurde somit journalistisch speziell betreut. Auch die finanzielle Ausstattung zeigt seine Bedeutung, denn von den 4.500 MDN, die die Sonderredaktion von Radio DDR für die Frühjahrsmesse 1966 insgesamt beantragte, entfielen 2.500 MDN auf die Sonderredaktion des Messe-„Werbefunks“.26 Es wurden also ca. $55 \%$ der Mittel zur Betreuung des „Werbefunks“ eingeplant. Das war jedoch eine Investition, die sich auszahlte, denn schon ab 8,3 Minuten verkaufter Sendezeit waren die Kosten für die Redaktion des „Werbefunks“ wieder eingespielt. Insgesamt brachte der „Werbefunk“ am Tag - falls die Preise so blieben - einen Betrag von 66.880 MDN ein. Es war also lohnenswert, politisch schwer zu begründende West-Werbung im Programm zu installieren, denn diese spielte an den sieben Messetagen 468.160 MDN ein.

Die „Internationale Werbesendung“ lief bis zur Frühjahrsmesse 1971 als eine in den Programmankündigungen separat ausgewiesene Sendereihe zwischen 7.33 und 8.30 Uhr auf Radio DDR I. Seit der Herbstmesse 1971 verschwand sie aus den Programmankündigungen. Nun liefen zwischen 8.05 und 8.30 Uhr die „Radiotips“.27 Das war jedoch nicht die einzige Veränderung, die im Frühjahr 1971 in der Hörfunklandschaft anlässlich der Leipziger Messe zu verzeichnen ist.

1971 wurde auch eine einheitliche Messeredaktion von Radio DDR und Berliner Rundfunk gebildet. Bisher hatten beide jeweils eigene Redaktionen unterhalten, die nun zusammengelegt wurden und in die sich auch der Deutschlandsender integrieren sollte. Da das bei dem für die DDR-Sender intern stets konstatierten ,Senderegoismus' eine schwierige Sache gewesen sein muss, war die finanzielle Seite hierbei vermutlich ein - wenngleich verspätetes - Entscheidungskriterium. Doch hatte die Umstrukturierung noch einen weiteren Grund. Denn neu war 1971 ebenfalls, dass es in den Planungen für das Programm von Radio DDR zur Messe einen Koordinator zwischen der Messe-Sonderredaktion der Berliner Zentrale und einer speziellen „Messewelle“ gab. ${ }^{28}$ Was war die „Messewelle“?

Nun, es hatte parallel zur Entwicklung in den zentralen Programmen auch eine Entwicklung im Regionalprogramm des Senders Leipzig gegeben, die 1971 zur Aufnahme einer speziellen „Messewelle“ führte. Ihr Ursprung ist ebenfalls Mitte der 1960er-Jahre zu finden, als der Hörfunk vermehrt dazu genutzt wurde, aus-

25 DRA, KV 16/66, 21. Januar 1966, Frühjahrsmesse 1966.

26 Ebd.

27 FF dabei 12/1971, S. 10; ebd. 37/1971, S. 10.

28 DRA, KV 5/71, 5. Februar 1971, Frühjahrsmesse 1971; KV 35/71, 20. Juli 1971, Herbstmesse 1971. 
ländische Partner anzusprechen. Seit 1964 gab es anlässlich der Messe selbst im Regionalprogramm aus Leipzig ein Extraangebot für die ausländischen Aussteller und Gäste: den „Messe-Service“.

\author{
II. Vorgeschichte der „Messewelle“ Teil 2 - \\ Radioprogramm zur Leipziger Messe im Regionalsender Leipzig
}

Innovatives Radioprogramm für wirtschaftlich wichtige Gäste der „Messe-Service“ des Regionalsenders Leipzig

Bereits in der Mitte der 1950er-Jahre wurde auch aus Leipzig über die Messe berichtet. 1956 übertrug der Regionalsender beispielsweise die 10-Minuten-Sendung „Leipziger Messereporter“, vermutlich auf Mittelwelle, die lokale Leipziger Fragen zum Inhalt hatte. ${ }^{29}$

Während der Frühjahrsmesse 1964 bot der Hörfunk aus Leipzig erstmalig einen UKW-Service, für den eine besondere UKW-Frequenz bereitgestellt wurde..$^{30} \mathrm{Es}$ war ein Service, der Gäste und Gastgeber mit den zur Messe notwendigen Informationen medial versorgte.

Aus der Messestadt kam damit der tontechnisch beste Hörfunk, den man aus der DDR empfangen konnte. Selbst im internationalen Vergleich war die DDR damit gut positioniert - ihr Hörfunk hatte mit den UKW-Übertragungen technisch das stets geforderte Weltniveau. Auch in anderer Hinsicht finden sich Innovationen des ostdeutschen Radioprogramms im Angebot aus Leipzig. Vom "Messe-Service“ wurden drei Mal täglich für 30 Minuten mehrsprachig praktische Hinweise zur Orientierung in der Messestadt und Empfeblungen für kulturelle Angebote ausgestrahlt. ${ }^{31} \mathrm{Um}$ die Übertragungen mit realem Stadtleben zu füllen, wurden Live-Schaltungen zum Wetteramt und Veranstaltungshinweise mit O-Tönen von Künstlern in den „Service“ integriert. ${ }^{32}$ Auch dies war durch die neue UKW-Technik besser als bisher möglich.

Im zweiten Jahr des „Messe-Service“ gab es auch wieder Originalsprechstellen, von denen aus die Menschen sich direkt an der Sendung beteiligen konnten. ${ }^{33}$ Dieser Publikumseinbezug war eine weitere Neuerung, die der Hörfunk der DDR

29 DRA, KV 6/56, 26. Januar1956, Frühjahrsmesse 1956.

30 Mitteldeutscher Rundfunk Leipzig, Verwaltungsarchiv, Dokumente 1924-1991 (im Folgenden: MDR), Sender Leipzig; 23. August 1989, Fakten zur Entwicklung der Messewelle des Senders Leipzig, Bl. 41.

31 MDR, 23. August 1989, Entwicklung der Messewelle, Bl. 41.

32 WeRnER KÖHLER, Erinnerungen an die Messewelle [Typoskript]. Bestand beim Verfasser. Ich bedanke mich bei Herrn Köhler für die Informationen!

33 Funkecho. Zeitung der Funkkorrespondenten des Sendebereichs Leipzig, Halle, Magdeburg 1/1965, 1. Februar 1965, o. p. [S. 2]. 
in den 1960er-Jahren durchführte. ${ }^{34}$ Und schließlich wurde im „Messe-Service“ 1965 erstmalig eine Stereo-Versuchssendung ausgestrahlt. ${ }^{35}$

Der „Messe-Service“ wurde 1965 von 7.30 bis 8.00, von 13.00 bis 14.00 und von 18.30 bis 18.55 Uhr übertragen. ${ }^{36} 1966$ berichtete man dann täglich schon um 6.30 und um 9.00 Uhr aus dem Pressezentrum über die wichtigsten Messeabschlüsse vom Vortage. Man hatte den „Service“ also weiter in den Morgen verlegt, da sich die Hörgewohnheiten für das Radio in dieser Hinsicht verändert hatten. Die Veränderungen in der Nutzung des Mediums waren ebenfalls erst seit den 1960erJahren im Programm umgesetzt.

In der Gesamtbetrachtung finden sich damit sämtliche Programminnovationen der 1960er-Jahre (und nicht nur die der DDR) im Sonderprogramm aus Leipzig. Doch bot der „Service“ auch in anderer Hinsicht Nützliches.

Schon ein Jahr nach ihrer Einführung auf Radio DDR I war 1966 auch im „Messe-Service“ aus Leipzig die West-Werbung präsent. Zwischen 7.30 und 8.40 Uhr wurde im Regionalprogramm zur besten Sendezeit die „Messe-Werbesendung“ von Radio DDR übernommen. Nach einer Grußsendung ab 8.40 Uhr wurde von 9.00 bis $9.30 \mathrm{Uhr}$ und von 13.00 bis 14.00 Uhr täglich der „Messe-Service“ übertragen. Daneben stellte man jeden Tag eine Preisfrage, die einen Bezug zur Messe hatte. Die Preise spendeten Betriebe aus dem Sendegebiet. Die Gewinner wurden am letzten Messetag in einer Originalübertragung [...] bekannt gegeben. ${ }^{37}$ Sowohl der Staat(-srundfunk) als auch die Menschen profitierten also direkt vom „Messe-Service“.

Im vierten Jahr des „Service“, zur Herbstmesse 1967, führte der ostdeutsche Hörfunk eine Umfrage durch, die angeblich mit überraschend starkem Echo nachwies, dass dieser Rundfunkkundendienst Hörbedürfnissen sebr entgegen kam. ${ }^{38}$ Hierzu finden sich keine Unterlagen mehr, sodass offen bleiben muss, welche Hörergruppe zu welchen Themen befragt worden ist. Jedenfalls wurde das Angebot weiter ausgebaut. Der „Service“ war ein Extra-Angebot - dieser Linie blieb man treu. Seit der Frühjahrsmesse 1968 lief auch ein „Verkehrsservice“ im Programm, der direkt aus einem Sonderstudio in der Verkebrsleitzentrale beim Volkspolizeikreisamt gesendet wurde. ${ }^{39}$ Es existierte eine Standleitung zwischen der Verkehrsleitzentrale der Polizei und dem Funkhaus, sodass sich der Reporter aus der Verkehrsleitzentrale ins laufende Programm einblenden konnte. Die „Messewelle“ war damit, auch gesamtdeutsch betrachtet, das erste deutsche Regional-

34 Christian KönNE, Hörfunk im Kalten Krieg. Berliner Radioprogramme in der Systemkonkurrenz, in: Michael Lemke (Hg.), Schaufenster der Systemkonkurrenz. Die Region Berlin-Brandenburg im Kalten Krieg, Köln 2006, S. 365-387, hier S. 372.

35 Wolfgang TieTz, Vier Jahrzehnte Rundfunkarbeit in Leipzig - Gedanken und Notizen, in: Beiträge zur Geschichte des Rundfunks 4/1987, S. 14-62, hier S. 45.

36 DRA, KV 119/1964, 6. Dezember 1964, Frühjahrsmesse 1965.

37 Funkecho. Zeitung für die ehrenamtlichen Korrespondenten des Sendebereichs Leipzig, Halle, Magdeburg (im Folgenden: Funkecho) 2/1966, 10. März 1966, o. p.

38 Zitiert nach: KöNNE, Ferienwelle und Messewelle (wie Anm. 16), S. 335.

39 TiETZ, Vier Jahrzehnte (wie Anm. 35), S. 45. 
programm, auf dem ein - zudem mehrsprachiger - „Verkehrsservice“ ausgestrahlt wurde. ${ }^{40}$ Das war so ungewöhnlich und Autoradios in der DDR so rar, dass in erster Linie die ausländischen Besucher durch Hinweisschilder an der Autobahn auf den „Verkehrsservice“ und seine Frequenzen aufmerksam gemacht wurden. ${ }^{41}$

Das Leipziger Angebot wurde aber nicht nur inhaltlich differenziert - es wurde auch zeitlich erweitert. Ende der 1960er-Jahre umfasste der „Messe-Service“ drei Mal 60 Minuten Sendezeit. ${ }^{42}$ Die tägliche Sendezeit war also verdoppelt worden. Im Frühjahr 1971 wurde der „Messe-Service“ abermals grundlegend verändert. Anlässlich der Messe gab es nun nicht mehr nur ein modernes Service-Angebot. Mit der „Radio-DDR-Messewelle“ konnte ein ganzes Sonderprogramm geboten werden. Erst 1971 waren hierfür die entscheidenden technischen und inbaltlichen Vorbereitungen abgeschlossen. ${ }^{43}$

\section{Die „Radio-DDR-Messewelle“.}

Grundfunktionen: Information für die Gäste und Unterbaltung für die Region

Wie ihr Vorgänger so war auch die „Messewelle“ als Service-Programm gestaltet.44 In der Zeit von 7.00 bis 19.00 , später bis 20.00 Uhr übertrug sie täglich ihr Programm, das am Samstag vor Messebeginn startete. Insgesamt verfügte die „Messewelle" damit an den acht Messetagen über 104 Sendestunden. ${ }^{45}$ Die Sendezeit war also um $333 \%$ gesteigert worden. Die in der Konzeption aufgeführten Programmbereiche ergeben folgende Anteile am 12-Stunden-Programm, die das vom SRK erstrebte Profil eines Informations- und Unterhaltungsprogramms deutlich abbilden: 46

40 In der Bundesrepublik wurde der erste regionale Verkehrsfunk im April 1971 ausgestrahlt. Die DDR nahm die ersten Verkehrsservice-Sendungen in ihren Programmen testweise 1975 auf. Forschungsergebnissen des Jahres 1975 zufolge wusste man, dass $31 \%$ aller zugelassenen PKW ein Autoradio besaßen. Weitere 28 \% der Autofahrer benutzten während der Fahrt ein Kofferradio. DRA, Bestand Soziologische Forschung, F 017-01-02/ 0028-0029, Bl. 33.

41 TiETZ, Erinnerungen (wie Anm. 5). Vgl. auch FF dabei 12/1971, S. 45.

42 Neue Deutsche Presse 18/1971, S. 6.

43 DRA, KV 5/71, 5. Februar 1971, Frühjahrsmesse 1971, Anlage Messewelle 22. Januar 1971.

44 DRA, KV 35/71, 20. Juli 1971, Herbstmesse 1971.

45 MDR, 23. August 1989, Entwicklung der Messewelle des Senders Leipzig, Bl. 41; FF dabei 12/1971, S. 46.

46 Die hier gezeigten Werte sind kein Widerspruch zum Musikanteil von $65 \%$, an den sich ehemalige Mitarbeiter erinnern, denn die Werbung und auch der Service waren immer mit Musik gemischt, deren genauer Anteil jedoch nicht mehr ermittelt werden kann. 


\begin{tabular}{|l|c|}
\hline \multicolumn{1}{|c|}{ Programmbereich } & Anteil am Programm in Prozent \\
\hline Nachrichten & 8,3 \\
\hline Service & 11,8 \\
\hline Werbung & 19,4 \\
\hline Unterhaltungs-Musik-Sendungen & 39,5 \\
\hline Wort-Unterhaltung & 4,1 \\
\hline
\end{tabular}

Hintergrund für die Einrichtung eines erweiterten Sonderprogramms war neben den Erfahrungen mit dem „Messe-Service“ unverändert die Bedeutung Leipzigs. Blieb die Stadt doch anlässlich der Messe ein wichtiger Faktor der Meinungsbildung für die vielen Tausend Besucher, Journalisten und sonstigen Vertreter, [...] für die Leipzig oft die DDR ist. ${ }^{47}$ Das galt es zu nutzen. Durch die "Messewelle“ wollte man die Repräsentanz der Messe und die Gastgeberfunktion der Stadt Leipzig wirkungsvoller unterstützen, als dies bisher durch den „Service“ möglich war. Das internationale Schaufenster Leipzig erhielt mit der „Messewelle“ eine zusätzliche Attraktion, die die Möglichkeit bot, die DDR und ihre Errungenschaften zeitlich erweitert und in Form eines Extraprogramms, also in einem politischmedial bedeutsamen Rahmen, dem Ausland zu präsentieren.

Und auch den „Werbefunk“, der bisher auf Radio DDR I gelaufen war, integrierte man in die „Messewelle“. Er wurde dort mehrsprachig übertragen. ${ }^{48}$ Der „Messe-Werbefunk“, den die Konzeption der „Messewelle“ abbildet, taucht später im offiziellen Programmschema nicht auf. Das ist bemerkenswert, denn immerhin entfielen auf ihn drei der elf Stunden Sendezeit, die die Konzeption ausweist. Mit dem Entstehen der „Messewelle“ 1971 lief gleichzeitig die „Internationale Werbesendung" auf Radio DDR I aus. Die Werbung wurde auf das Sonderprogramm aus Leipzig konzentriert. Dies passte in die politische Großwetterlage: In den 1970er-Jahren ging die DDR unter der Führung von Erich Honecker zur sogenannten Hauptaufgabe über. Ein sich selbst finanzierender Sozialismus war nicht erreicht worden. Es ging nun um den geliehenen, kleinen Wohlstand im Lande. Wirtschaftliche Versuche - auch im Hörfunk - waren damit passé. ${ }^{49}$

Bei der Gestaltung des Programms der „Messewelle“ wurden die mehrsprachigen Kundendienstinformationen mit Ausnahme des „Werbefunks“ und die unterhaltenden Sendungen vom Regionalsender und seinen Mitarbeitern gestaltet. ${ }^{50}$ Die Nachrichten und der „Werbefunk“ verblieben bei der Zentrale.

47 MDR, o. D. (vermutlich 1969/70), Radio DDR - Sender Leipzig, Direktor, Die Radio-DDR-Messewelle (Vorlage für Radio DDR) (im Folgenden: Konzeption Messewelle), Bl. 1.

4840 Jahre academixer - unser Buch, Leipzig 2007, S. 21.

49 STEINer, Von Plan zu Plan (wie Anm. 8), S. 187-223.

50 MDR, o. D., Konzeption Messewelle, Bl. 2. 


\section{Die Sendeinhalte}

Informationen. Leipzig als sozialistischer Wirtschafts- und Kulturstandort

Informationen hörte man in der „Messewelle“ über die Messe und die Stadt - für die Besucher und die Leipziger. Der „Messe-Service“ übertrug als Magazinsendung auch innerhalb der Messewelle wie seit 1964 drei Mal am Tage, aber nun wieder nur für eine halbe Stunde, konzentriert mehrsprachige Kundendienstinformationen, in denen Verkehr, Wetter, Veranstaltungen und Dienstleistungen enthalten waren. Daneben wurden unverändert kurze Gespräche und Reportageausschnitte [...], sowie [...] Originaleinblendungen aus dem Zentrum, Leipzig Information' ausgestrahlt. ${ }^{51}$ Die Informationen hörte man anfänglich in drei, später in fünf Fremdsprachen. ${ }^{52}$

Damit war die „Messewelle“ wesentlicher Bestandteil der Messeberichterstattung für Kaufleute, Experten, Außenbändler, Besucher und Gastgeber. ${ }^{33}$ Hier folgte das Programm aus Leipzig dem IUP-Konzept (Informations- und Unterhaltungsprogramm) von Radio DDR, das seit Ende der 1960er-Jahre Dienstleistungen als eine bestimmende Sendeform für die Programme von Radio DDR vorsah. ${ }^{54}$ Das galt auch für seine Sonderprogramme „Ferienwelle“ und „Messewelle" sowie für die Regionalsender allgemein. Mit Kundendienstinformationen, Messewerbefunk, Informationen über Verkebrssituation, Veranstaltungen, Messeformalitäten, individuelle Dienstleistungen, Wetter usw. war der Servicecharakter der „Messewelle“ für das SRK erfüllt. 55

Als zweiter Informationsteil mit dem Schwerpunkt Messe wurden die „Messeneuigkeiten" einmal täglich mit 15 Minuten Sendezeit übertragen. Sie berichteten vom Tagesgeschehen auf der Messe. ${ }^{56}$

Die Reihe „An der Messe gemessen ...“ zeigte entsprechend der aktuellen Messeargumentation und in Abstimmung mit der, Messerevue' auf Radio DDR I die ökonomischen Errungenschaften der DDR, sowie die wirtschaftliche Zusammenarbeit mit den anderen sozialistischen Ländern auf. „An der Messe gemessen ..." hatte folgende Bestandteile: Das „Messegespräch mit Produzenten“ war eine Konferenzschaltung zwischen Messestand und den Produzenten, in der Betriebe der DDR zu Wort kamen. In ihm wurde anlässlich der Auszeichnung von DDRBetrieben der Zusammenhang zwischen Messeerfolg und der Arbeit des Betriebskollektivs ins Blickfeld gerückt und besonders verdiente Kollektive vorgestellt. Beim „Messebummel mit Spezialisten“ begleitete die „Messewelle“ Fachleute auf

51 Ebd., Bl. 7.

52 TiETZ, Vier Jahrzehnte (wie Anm. 35), S. 45.

53 DRA, KV 9/73, 12. Februar 1973, Messesonderredaktion.

54 Neue Deutsche Presse 18/1971, S. 6 f., hier S. 7.

55 DRA, KV 5/71, 5. Februar 1971, Frühjahrsmesse 1971, Anlage zur Messewelle vom 22. Januar 1971.

56 TIETZ, Erinnerungen (wie Anm. 5). 
ihren jeweiligen Spezialmessen. Im „Messetreff mit Handelspartnern“ wurden die Handelsbeziebungen zu unseren sozialistischen Bruderländern und anderen Partnern vorgestellt, wobei die Rolle Leipzigs als Mittler und Förderer des internationalen Handelns zu betonen war. Wer hören wollte, wie die idealisierte Planwirtschaft arbeitete, konnte sich hier eine akustische Kostprobe verschaffen.

$\mathrm{Zu}$ den Informationen über Wirtschaft, Handel und internationale Kooperation traten diejenigen über die Messestadt. Die Sendefolge „Messemann auf Stadtrundgang" bildete in vorproduzierten Beiträgen Leipzig als sozialistische Großstadt der $D D R$ ab, die nicht nur weltweit bekannter Handelsplatz, sondern eine Stadt mit ausgeprägtem geistig-kulturellem Profil war. Hier wurden Institutionen und Einrichtungen vorgestellt, die als für das gesellschaftliche Leben der Stadt [...] repräsentativ angesehen wurden. ${ }^{57}$ Die Stadt Leipzig mit ihren sozialistischen Errungenschaften und kulturellen Monumenten sollte vorgestellt werden. Der „Messemann“" war das Maskottchen der „Messewelle“, das vom Schöpfer des „Sandmanns“, Gerhard Behrend, stammte. In den Erinnerungen ehemaliger Mitarbeiter hatte der „Messemann“ eine andere Ausrichtung. Hier berichtete er über amüsante Nebensächlichkeiten rund ums Messegeschehen. Ein realer „Messemann" fuhr mit dem Übertragungswagen durch Leipzig und berichtete beispielsweise live über Messejubilare, private Messevermieter, neue gastronomische Einrichtungen, unterbielt sich mit fliegenden Händlern, Straßenmusikanten, befragte Messegäste etc.

Der Bereich der Information war also so angelegt, dass den ausländischen Gästen die Informationen über die Stadt, Messe, Land und System propagandistisch aufgeladen präsentiert wurden. Die Leipziger Bevölkerung konnte die übliche (Medien-)Politik wiedererkennen sowie ihre Stadt unter einem anderen Betrachtungswinkel wahrnehmen.

Neben diesen im weiteren Sinne informatorischen Teilen waren mit der Vergrößerung des Programmangebots 1971 zur „Messewelle“ die Unterhaltungsanteile vergrößert worden. ${ }^{58}$

\section{Die Sendeinhalte - Unterhaltung für die Messestadt}

Die Unterhaltung fand ihren Platz im Programm mit Musik, Gruß- und Wunschsendungen sowie dem Preisausschreiben, das um 8.30, 14.30 und 18.15 Uhr lief.59 An den unterhaltenden Sendungen konnten sich die Hörer schriftlich oder telefonisch beteiligen. 60

57 MDR, o. D. Konzeption Messewelle, Bl. 7.

58 Nachweise in: KönNE, Ferienwelle und Messewelle (wie Anm. 16), S. 336 f. Vgl. hierzu auch: CHRISTIAN KÖNNE, Wirtschaftssendungen für die DDR. Hörfunk zur Finanzierung des Sozialismus, in: Deutschlandarchiv. Zeitschrift für das vereinigte Deutschland 44 (2011), Nr. 2, S. 222-229, hier S. 227 f.

59 MDR, Werbematerial zur Messewelle, Bl. 43.

60 MDR, 23. August 1989, Entwicklung der Messewelle, Bl. 41. 
Die musikalische Unterhaltung war auch in der „Messewelle“ zentral. Der Musikanteil am Programm lag bei etwa 65 Prozent. Ein ehemaliger Redakteur der "Messewelle“ gibt in seinen Erinnerungen an, dass diese ausschließlich so genannte $U$-Musik, speziell Schlager und Jazz, übertrug. ${ }^{61}$ Zur Messe wurde dabei das sonst vorgeschriebene 60/40-Verbältnis [...] außer Kraft gesetzt und in etwa umgedreht. ${ }^{62}$ Entsprechend ihres weltgewandten Charakters war die „Messewelle“ ein Programm mit „einem hohen Anteil internationaler Musik“ “. ${ }^{63}$ Hier kamen strategischer Weise im Interesse unserer nationalen und internationalen Bedeutung [...] Künstler aus dem kapitalistischen Ausland ins Programm. ${ }^{64}$ Die Leipziger Messe war ein internationaler Handelsplatz - selbst die Hörfunkmusikgestaltung hatte dies abzubilden.

Der „Messemusikbasar“ bot Messegästen und Gastgebern die Möglichkeit, Grüße und Glückwünsche zu übermitteln. Gleichzeitig sollten hier besondere Verdienste um die Messe gewürdigt werden. Die Grüße und Glückwünsche wurden in Originaleinblendungen [...] direkt aufgegeben, um [...] in diese Sendung Messeatmosphäre hineinzutragen und Informationen zu vermitteln. ${ }^{5}$ Die Grüße hatten aber auch Potenzial für Messe und Sozialismus. Die Wirkungsmacht, die Grüßen zugeschrieben wurde, war groß. Neben den propagandistischen Effekten für die Gäste wurde vor allem eine gegenseitige Erziehung der Menschen hin zu sozialistischem Verhalten sowie die Ausbildung eines Heimatbewusstseins durch sie vermutet. ${ }^{66}$ Sie sollten also zur Erschaffung der neuen Gesellschaft und der sozialistischen Nation in Abgrenzung zur Bundesrepublik beitragen.

Als Übernahme von Radio DDR I lief die „Messe-Revue“, in der neben Musik auch Interviews zum Tagesgeschehen mit einem Mitarbeiter des Messeamtes übertragen wurden. Zur Unterhaltung mit Musik trat jene im Wort. Auch sie bildete, wie vorgegeben, die sozialistische Metropole Leipzig ab. Hierzu legte die Konzeption fest: Im Zusammenhang mit den unterhaltenden Musiksendungen werden feuilletonistische Beiträge gestaltet, die das internationale Musikleben der Messestadt widerspiegeln, bzw. historische Informationen, vor allem über die [...] sozialistische Geschichte der Messestadt Leipzig vermitteln. ${ }^{67}$

61 KöHLER, Erinnerungen (wie Anm. 32). Tietz meint ebenfalls, dass der Anteil der Westmusik bei „über $50 \%$ “lag.

62 KÖHLER, Erinnerungen (wie Anm. 32).

6340 Jahre academixer (wie Anm. 48), S. 21. Ich bedanke mich bei den „academixern“, speziell bei Christian Becher, für die Informationen und die Nutzung ihres Archivmaterials!

64 Zitiert: KönNE, Ferienwelle und Messewelle (wie Anm. 16), S. 337.

65 MDR, o. D., Konzeption Messewelle, Bl. 8.

66 Christian Könne, Die Radio-DDR-Ferienwelle. Programm für Urlaub im Sozialismus, in: Rundfunk und Geschichte 35 (2009), H. 3/4, S. 15-29, hier S. 20 f.

67 DRA, KV 5/71, 5. Februar 1971, Frühjahrsmesse 1971, Anlage Messewelle, 22. Januar 1971. Der Vergleich mit den Ankündigungen in der „FF dabei“ und einem als Postkarte öffentlich ausgegebenen, aber groben Programmschema aus dem Jahre 1974 zeigt weiterhin, dass die Konzeption in großen Teilen später so über den Sender ging. FF dabei 12/1971, S. 46. Für die Herbstmesse 1971 findet sich das Schema etwa in gleicher Form, allein der 
Die Sendefolge „Roberts Raritätenmesse“ brachte den Menschen, den Vorgaben folgend, die Stadtgeschichte sowie die Gegenwart der sozialistischen Handelsmetropole in sebr aufgelockerter Form nahe. Die einzelnen Folgen wurden für einen Erzäbler geschrieben und mit Szenen verbunden. Inhaltliche Basis waren alte Leipziger Marktzeitschriften und Dokumente aus dem Museum für Stadtgeschichte. 68 Präsentiert wurden hier Kuriosa aus der vielbundertjäbrigen Messehistorie, die vom Schauspieler Hans-Robert Wille (Namensgeber) auf vergnügliche Weise an den Hörer gebracht wurden. ${ }^{69}$

\section{Programmveränderungen für die Leipziger}

\section{Unterhaltung auf Sächsisch - Gründe und Inhalte einer Konzeptionswandlung}

In der „Messewelle“ wurde auch die Reihe „Schnurz und Biebe“, die von Beginn an zum speziellen Klang der „Messewelle“ beitrug, fortgeführt. Die beiden Figuren waren bereits seit 1965 als [...] Sächsisch-Sprachlebrer im Programm und gaben dort ihre „Sächsisch im Funk“-Lektionen. ${ }^{70}$ Es handelte sich um etwa drei Minuten lange Folgen, in denen „Schnurz“ und „Biebe“ den Menschen sächsische Begriffe und Besonderheiten erläuterten. An dieser Sendereihe konnten sich die Hörer durch Einsendung von Vorschlägen für typische Redensarten oder sächsischem Vokabular beteiligen. ${ }^{71}$ Zwar lief „Sächsisch im Funk“ schon seit 1965, doch wurde es erst in den Programmankündigungen des Jahres 1974 abgedruckt. Dass die Reihe bis dato unerwähnt blieb, lag vermutlich an den Bedenken der Programmverantwortlichen mit sächsischen Mundartprogrammen politische Schwierigkeiten auf sich zu ziehen, da sie eine sprachliche Verbindung zu Walter Ulbricht aufzeigten. ${ }^{72}$ Ulbricht war Leipziger und seine sprachliche Dialektfärbung war unter der Hand stets für eine Verballhornung der DDR-Funktionärssprache gut. Die Reihe zeigte von Beginn an, dass humorige Beiträge auf Sächsisch bei den Menschen ankamen. Dementsprechend wurde „Schnurz und Biebe“ in die „Messewelle“ integriert und auch für die „Ferienwelle“ vom Sender Rostock verwendet. ${ }^{73}$

Mit der Etablierung der „Messewelle“ und der damit erweiterten Sendezeit wurde nach diesen Erfahrungen nicht nur der Anteil der Unterhaltung vergrößert, sondern diese auch in verstärktem Maße mit sächsischem Dialekt versehen. Die Redaktion des Regionalsenders begann hierzu eine Kooperation mit den „acade-

Werbefunk fehlt. DRA, KV 35/71, 20. Juli 1971, Herbstmesse 1971; KV 7/72, 24. Januar 1972, Frühjahrsmesse 1972.

68 MDR, o. D., Konzeption Messewelle, Bl. 8.

69 KÖHLER, Erinnerungen (wie Anm. 32).

70 MDR, 1990, Sachsen Radio, Messewelle, Bl. 2.

71 KÖHLER, Erinnerungen (wie Anm. 32). TIETZ, Erinnerungen (wie Anm. 5).

7240 Jahre academixer (wie Anm. 48), S. 19.

73 KÖHLER, Erinnerungen (wie Anm. 32). 
mixern“, da sie in der Springerstraße neben der „Pfeffermühle“ als das beliebteste Leipziger Kabarett galten. Zudem war [...] durch jabrelange Zusammenarbeit bekannt, dass alle männlichen Mitglieder sich bereits als Autoren bewährt hatten. ${ }^{74}$ Ebenso wichtig war jedoch, dass die „academixer" Kabarett auf Sächsisch machten. Denn anders als noch in den 1950er-Jahren erhoffte sich die SED inzwischen durch Mundartprogramme eine stärkere Identifikation mit der DDR. ${ }^{75}$ Dies dürfte ein wichtiges Argument für die sprachliche Ausrichtung der „Messewelle“ auf das Sächsische sein. Der Unterhaltungsanteil des Programms fokussierte zunehmend die sächsische Bevölkerung. Damit entsprach der Sender Leipzig in seiner inhaltlichen Entwicklung den Parteivorgaben, die für die Regionalsender seit Mitte der 1960er-Jahre forderten, das sozialistische Heimatgefübl zu wecken, das für das Staatsbewußtsein wichtig ist. ${ }^{76}$ Nach diesem Konzept arbeitete auch die „Messewelle“. Die Messe wurde für die Leipziger Bevölkerung zum regionalen Highlight des Hörfunks in sächsischer Mundart umgestaltet. Die lokalen Ereignisse, die in den Bezirksprogrammen von Radio DDR übertragen wurden, wertete man dabei als Belege der Gesamtentwicklung hin zur sozialistischen Gesellschaft. ${ }^{77}$

Die „academixer" gestalteten in der "Messewelle“ die Reihe „aMESSEments“. Diese liefen täglich von 16.30 bis $17.00 \mathrm{Uhr}$ und übernahmen damit den Sendeplatz von „Roberts Raritätenmesse“. Die Historie mit wirtschaftlichem und sozialistischem Fokus wurde von einem Unterhaltungsprogramm mit sächsischem Zungenschlag abgelöst. Für die Bewohner der Messestadt wurden am frühen Abend Witze und Witzchen, nicht ernst zu nehmendes Aktuelles und Historisches, sächsischer Humor und Poesie geboten, wobei die Ansage für die „aMESSEments“ vom Hörfunk aus unmissverständlich war: Ulk, Nonsens und Blödelei so viel wie möglich, aber - kein politisches Kabarett auf dem Sender. ${ }^{78}$ Die Lieder, Gedichte und Geschichten „aMESSEments“ waren vorproduzierte Beiträge, die seit 1976 übertragen wurden. ${ }^{79}$ Die musikalische Gestaltung übernahm ein Redakteur des Regionalsenders. ${ }^{80}$ Kurz vor der ersten Staffel wurde klar, dass zusätzlich ein Moderator für die Übergänge notwendig war. So kamen die Kabarettisten bemerkenswerter Weise live ans Mikrofon, obne dass die Texte zuvor zur Abnabme vorgelegt werden mussten.

74 Ebd.

75 KÖNNE, Ferienwelle und Messewelle (wie Anm. 16), S. 338 f.

76 BArch, SAPMO, DY 30/IV A 2/9.02/66, 25. Januar 1967, Bericht des SRK vor dem Sekretariat des ZK.

77 Willi LaNGe, Blickpunkt Heimatsender. Über den kulturellen und ethischen Auftrag der Bezirkssender von Radio DDR (Rundfunkjournalistik in Theorie und Praxis, Sonderheft), Berlin (Ost) 1966, S. 49.

78 Zitiert nach: KöNNE, Ferienwelle und Messewelle (wie Anm. 16), S. 338 f.

79 MDR, 1990, Sachsen Radio, Messewelle, Bl. 2; Köhler gibt in seinen Erinnerungen 1978 als Sendebeginn an.

80 KÖHLER, Erinnerungen (wie Anm. 32). 
Die „academixer" entwickelten für ihre Halbstundenreihe verschiedene Beiträge, darunter beispielsweise Ulkinterviews über seltene Hobbys von Leipzigern oder abstruse Betrachtungen über die Entstehung der Namen von Leipziger Stadtteilen. Die Beiträge in den „aMESSEments“ waren „Sächsische Witze“, „Stimmt denn das?“, „Das Interview“, die „Leipziger Nachrichten“, „Leipziger Sehenswürdigkeiten“ oder der „Chronikonkel“. 81

Ende der 1970er-Jahre wurde die „Sächsische Hitparade“ Teil der „aMESSE ments“, die am ersten Messesonntag jeweils sechs Titel der „academixer" vorstellte, von denen täglich einer wiederholt wurde. ${ }^{82}$ Durch Anrufe und Zuschriften wurde für den letzten Messetag der Sieger dieser Konkurrenz ermittelt. Dieser führte in der nächsten Messe den Ausscheid gegen fünf neue Lieder an, selbst bei Wiederwahl jedoch höchstens drei Mal. Die meisten Lieder hatten einen Leipzigbezug wie beispielsweise „Bahnhofsblues“ oder „Middernachd in Leibzsch“. Der bekannteste Song der „Sächsischen Hitparade“ ist vermutlich „Sing, mei Saggse, sing" von Jürgen Hart. Für die „Sächsische Hitparade“ wurden bis 1990 etwa 100 Titel produziert. Sie war ein großer Erfolg bei der Bevölkerung, die sich mit angeblich etwa 1.500 Postkarten beziehungsweise Anrufen während der Messe an der Abstimmung beteiligte..$^{83}$

In der Mitte der 1980er-Jahre starteten die "academixer" die Familienserie „Bädzold - 3 mal bläkn“. Sie war die erste Comedyserie der sächsischen Rundfunkgeschichte überhaupt, in der [...] in fortlaufenden Episoden hanebüchene Alltags-Erlebnisse einer Leipziger Familie dargeboten wurden. ${ }^{84}$ Die Pätzolds - wie sie Außer-Sächsisch hießen - waren der Vater Rudi, der als Lagerverwalter im Verschiebebahnhof Nord arbeitete, die Mutter Irma, die Aushilfsverkäuferin in der Bäckerei Born war und die Tochter Ramona, die als Schülerin die 9. Klasse besuchte. Sie wohnten seit Jabren in der elterlichen Wohnung, 4. Stock rechts in der Lausener Straße. Die Klingel ist seit 1947 kaputt, deshalb muss man, wenn man zu ibnen will: 3 mal bläken". ${ }^{85}$ Hier wurde eine typische Familie im Sozialismus gezeigt. Schon durch den Titel war die Serie als eine Anspielung auf die Reihe „Neumann - 2 mal klingen“ gekennzeichnet, die seit 1968 auf Radio DDR I lief und eine typische sozialistische Vorzeigefamilie darstellte, die als Vorbild und Orientierung für die Menschen dienen sollte. ${ }^{86}$ Während im Zentralprogramm ein ideales sozialistisches Leben vorgestellt wurde, wie es sich die SED für die Menschen in der DDR wünschte, war im Regionalprogramm Lachen über ein realsozialistisches Leben möglich und erwünscht. War dies eine Wandlung in der Ver-

8140 Jahre academixer (wie Anm. 48), S. 22 f.

8210 Jahre Sächsische Hitparade, in: Leipziger Volkszeitung, 13. März 1989 [zitiert nach: academixer Archiv, Presse].

83 KÖHLER, Erinnerungen (wie Anm. 32).

8440 Jahre academixer (wie Anm. 48), S. 22.

85 Academixer Archiv, Schriftgut „Bätzold - 3 mal bläkn“, o. D., Skript zur 1. Folge.

86 BArch, DR 6-610, 19. November 1969, Bericht über Ergebnisse bei der Durchführung des Maßnahmenplans über „Die Aufgaben der Kultur bei der Entwicklung der sozialistischen Menschengemeinschaft". 
wendung von Unterhaltung? Diese hatte seit Mitte der 1960er-Jahre der Bildung im Sozialismus gedient und eben auch der Ausbildung ,sozialistischen Verhaltens‘. War man von diesem Konzept abgegangen und hatte die Unterhaltung - in den regionalen Programmen - seit den 1980er-Jahren vor allem als Instrument zur Thematisierung und damit Kanalisation von Frustrationen eingesetzt? Jedenfalls sonderte sich die Region Leipzig hier vom Zentralstaat und -programm ab. Doch gleichzeitig waren die bei den „Bädzolds“ erzählten Erlebnisse authentisch, sie waren etwas, das es so in der Bundesrepublik nicht gab. Mit ihrem originalen wie originellen sozialistischen Familienleben wurden die „Bädzolds“ auch in der „Ferienwelle“ übertragen. ${ }^{87}$

Bis zur Frühjahrsmesse 1990 wurden insgesamt 192 „aMESSEments“ ausgestrahlt. ${ }^{88}$ Nach der ,Wende` liefen sie bis 1991 beim Nachfolgesender Sachsenradio weiter.

\section{Programmveränderungen für die Gäste}

Die Wiederaufnahme der Werbung -

Beiträge für den Sozialismus auf Kosten der Information der Gäste

Noch eine weitere Veränderung erfolgte in den 1980er-Jahren. Sie betraf den Service und die Werbung, also den Teil des Programms für die ausländischen Gäste. Ebenso unvermittelt, wie die „Werbesendung“ in den 1970er-Jahren verschwand, tauchte sie in der Mitte der 1980er-Jahre im Programm aus Leipzig erneut auf. 89 Ohne dass sich in den Komiteevorlagen Gründe für die Wiederaufnahme finden, gab es in der Planung für die Leipziger Herbstmesse 1984 ein Kollektiv „Werbefunk", das fünf Personen umfasste. Die Anzahl ist insofern bemerkenswert, als im Vergleich hierzu die Kollektive Internationale Verbindungen aus drei, Radio DDR aus zwölf und Berliner Rundfunk aus fünf Personen bestanden. Die personelle Ausstattung der Werbung war zum zweiten Start also vergleichsweise üppig. Die Summe für die Bewirtschaftung der Messekollektive blieb - wie seit den 1970erJahren - mit 2.600 M nahezu unverändert. ${ }^{90}$ Hiervon war der „Werbefunk“ jedoch offenbar ausgenommen, denn die Erfassung aller Ausgaben und Einnabmen zur Vorbereitung und Realisierung von Werbesendungen wurde gemäß einer Vereinbarung der HA [= Hauptabteilung] Planung/Ökonomie während der Herbstmesse über eine separate Kostenstelle vorgenommen."91 Auch dies spricht für die Bedeutung, die man dem „Werbefunk“ innerhalb der „Messewelle“ zusprach.

87 KÖNNE, Radio-DDR-Ferienwelle (wie Anm. 66), hier S. 23.

8840 Jahre academixer (wie Anm. 48), S. 23.

89 DRA, KV 4/84, o. D., Frühjahrsmesse 1984. Hier wird nicht erwähnt, dass der „Messewerbefunk“" wieder eingeführt wird.

90 DRA, KV 3/89, o. D. Frühjahrsmesse. Vgl. auch KV 25/89, o. D. Herbstmesse 1989.

91 DRA, KV 14/84, o. D., Herbstmesse 1984. Die übrigen Ausgaben wurden mit 2.600 $\mathrm{M}$ festgesetzt, sie waren also nahezu unverändert geblieben. 
Für die Revitalisierung des „Werbefunks“ dürften wiederum die finanziellen Schwierigkeiten der DDR verantwortlich sein, die Mitte der 1980er-Jahre beispielsweise zur Gewährung der beiden Milliardenkredite durch die Bundesrepublik führten. Die SED war erneut gezwungen, die politische Linie zugunsten von finanziellen Transfers zu verändern. Für das Jahr 1985 findet sich keine Information zur Werbung anlässlich der Messen in Leipzig. Seit der Frühjahrsmesse 1986 wurde dann im Interesse einer geschäftsfördernden Messeatmosphäre [...] entsprechend Ministerratsbeschluß - auf der Messewelle [...] jeweils ab $8.00 \mathrm{Ubr}$ und 16.00 Ubr und am 22. März ab $8.00 \mathrm{Ubr}$ [die Reibe] "Messewerbung international" ausgestrablt, die vom Kollektiv Messewerbefunk gestaltet wurde. ${ }^{92}$ Doch nicht nur das. Die Mitarbeiterzahl des Kollektivs des „Werbefunks“ war seit 1984 verdoppelt worden. Dieses umfasste 1986 elf Mitarbeiter. Damit war die Versorgung und Organisation der Werbung für die ausländischen Kunden gut aufgestellt. Denn das Messe-Kollektiv von Radio DDR umfasste ebenfalls nur elf, das des Berliner Rundfunks sogar nur neun Mitarbeiter. ${ }^{93}$

Die Bedeutung des „Werbefunks“ nahm weiter zu. So wurde im Folgejahr entschieden, dass in die Früb-Werbesendungen [...] bei aktuellem Bedarf Verkebrsinformationen und in dringenden Fällen Service-Informationen für Messegäste jeweils nach Abstimmung mit dem Leiter des Kollektivs Messewerbefunk eingeblendet werden. ${ }^{94}$ Der „Service“ als ursprüngliches Zentrum der „Messewelle“ war im Hinblick auf das Programm für die Messegäste auf den zweiten Platz gerutscht. Zwar sollte er aktuell bleiben, doch dass die Werbung ihr Einverständnis zur Übertragung aktueller Service-Leistungen geben musste, zeigt deren Bedeutung und wirft die Frage nach der tatsächlichen Aktualität des „Service“ auf. Das zentrale Programmelement für die ausländischen Gäste war jetzt deren Werbung. ${ }^{95}$ Mit der Herbstmesse 1987 wurde die Sendezeit des „Werbefunks“ in der wichtigen Zeit am Morgen nochmals um 30 Minuten erweitert. Die Werbung lief nun, wie in den 1970er-Jahren, bereits ab 7.30 Uhr im Programm. ${ }^{96}$ Auch was die Produktionszeiten anging, hatte sich die Werbung während der Leipziger Messe einen soliden Platz verschafft. So war für die Produktion der Werbebeiträge [...] von der Studiotechnik Rundfunk, zu sichern, dass täglich elf Stunden für die Produktion der Werbebeiträge zur Verfügung standen. ${ }^{97}$ Im Gegensatz zu den 1970erJahren findet sich in den Unterlagen der 1980er-Jahre keine Konzeption, die den

92 KV 7/86, o. D., Frühjahrsmesse 1986. KV 25/86, o. D., Herbstmesse 1986.

93 DRA, KV 4/85, o. D., Frühjahrsmesse 1985. Hier umfasste das Kollektiv des „Werbefunks" fünf Personen. Es finden sich keine weiteren Hinweise auf die Ausgestaltung. Die KV 27/85, o. D., Herbstmesse 1985, nennt für das Kollektiv des „Werbefunks“ 13 Mitarbeiter.

94 DRA, KV 6/87, o. D., Frühjahrsmesse 1987.

951987 hatten die Kollektive folgende Größe: acht Mitarbeiter Werbefunk, acht Radio DDR, sechs Berliner Rundfunk.

96 DRA, KV 29/87, o. D., Herbstmesse 1987. Ob es auch am Nachmittag Werbung gab, kann nicht ausgesagt werden.

97 DRA, KV 6/87, o. D., Frühjahrsmesse 1987. 
Programmablauf der „Messewelle“ zeigt. Es finden sich auch keine Preislisten, die für den Werbefunk galten. Auch stellt sich die Frage, welche Produkte im Werbefunk beworben worden sind. Ein ehemaliger Mitarbeiter erinnert sich für die späteren Jahre der „Messewelle“ an einen „Werbefunk“, der DDR-Konsumgüter bewarb. ${ }^{98}$ Doch warum sollten westliche Firmen DDR-Konsumgüterwerbung finanzieren?

Mit den gezeigten Veränderungen hatten sich die Programmanteile der „Messewelle" bis zur Mitte der 1980er-Jahre wie folgt entwickelt: 99

\begin{tabular}{|l|c|c|}
\hline \multicolumn{1}{|c|}{ Programmbereich } & $\begin{array}{c}\text { Anteil am Programm } \\
\text { in Prozent }\end{array}$ & $\begin{array}{c}\text { Veränderung } \\
\text { in Prozent }\end{array}$ \\
\hline Nachrichten & $7,0 \%$ & $-1,3$ \\
\hline Service/Werbung & $26,9 \%$ & $-4,3$ \\
\hline Unterhaltungs-Musik-Sendungen & $48,7 \%$ & $+9,2$ \\
\hline Wort-Unterhaltung & $3,8 \%$ & $-0,3$ \\
\hline
\end{tabular}

Die „Messewerbung International“ findet sich in den Programmankündigungen für die ostdeutsche Bevölkerung nicht. Ebenso fehlt 1986 der Hinweis auf die nur bis 1984 angekündigte Sendereihe „Sächsisch im Funk“.100 Doch auch im ausgewiesenen Schema ist für die in den internen Dokumenten nachgewiesene „Internationale Werbesendung" genügend Sendezeit zu erkennen. Der „Werbefunk“ blieb also wirtschaftlich so bedeutsam wie politisch heikel. Gleichzeitig zeigt sich die Zunahme des Unterhaltungsanteils der „Messewelle“. Die inhaltlich dargestellte Entwicklung ist damit auch in den Programmzeiten nachzuweisen.

\section{Sendezeit und Sendefrequenzen - die „Messerwelle“: ein Programm für die Region Leipzig}

Da eine Versorgung durch genügend Strahler mit ausreichender Kapazität für das Senden von Hörfunkprogrammen in der DDR bis in die 1970er-Jahre ein großes Problem war, wurde dem Sender Leipzig für die „Messewelle“ ein mobiler MWStrahler zur Verfügung gestellt. ${ }^{101}$ Es ist zu fragen, ob der Beginn der „Messe-

98 KÖHLER, Erinnerungen (wie Anm. 32). Tietz sagt in seinen Erinnerungen, dass überhaupt keine Werbung gemacht wurde.

99 FF dabei 36/1986, S. 11 f. Als Angaben zur Berechnung liegen in der FF dabei vor: 7.30 / 12.30 / 17.30 Uhr „Messe-Service“; 9.05 / 14.05 Uhr „Messestadt-Notizen“; 11.00 / 19.10 Uhr „Messe-Musikmann“; 9.15 / 12.15 / 14.15 / 17.15 / 19.15 Uhr „Preisausschreiben“; 10.30 Uhr „Schlagermuseum“; 18.00 Uhr „Amessements“; 18.30 Uhr „Messereport“. In dieser müssen Service und Werbung zusammen ausgewiesen werden, da sich keine getrennte Aufstellung der Sendezeiten mehr findet. Um die Veränderung zu beschreiben, wurden beide Werte der ersten Tabelle addiert.

100 FF dabei 36/1984, S. 37.

101 MDR, o. D., Konzeption Messewelle, Bl. 2. Tietz spricht von einem mobilen MWsowie einem mobilen UKW-Strahler. TIETZ, Vier Jahrzehnte (wie Anm. 35), S. 46. 
welle“ im Jahre 1971 deshalb erfolgte, weil erst zu dieser Zeit die funktechnischen Gegebenheiten für ein eigenes Sonderprogramm vorhanden waren. Die technische Entwicklung in anderen Bereichen legt dies nahe. Der mobile MW-Strahler hatte seinen Standort in Markkleeberg und wurde nach der Messe für andere Zwecke wieder abgebaut. Seine Leistung betrug $5 \mathrm{~kW}$. Daneben wurde die „Messewelle“ zunächst über UKW Leipzig 88,45 und $93,85 \mathrm{MHz}$ ausgestrahlt. ${ }^{102}$ Bis zur Herbstmesse 1971 hatten sich folgende Veränderungen ergeben: Nun konnte die „Messewelle“ vormittags täglich über die Frequenzen UKW Leipzig IV (93,85 MHz), UKW Brocken IV (94,6 MHz), UKW Dequede IV (94,9 MHz) und auf der Mittelwelle $557 \mathrm{kHz}$ in der Zeit von 7.00 bis $10.00 \mathrm{Uhr}$ sowie nachfolgend zwischen 10.00 und 18.55 Uhr unverändert über die Frequenz MW $557 \mathrm{kHz}$ und zusätzlich über UKW Markkleeberg 89,3 MHz abgestrahlt werden. ${ }^{103}$

Eine Untersuchung des Programms der Studiotechnik Funkhaus Leipzig und des Funkamts Leipzig hatte am 4. Dezember 1970 folgende Reichweite ergeben, die als optimal gekennzeichnet war: Im Norden hatte man eine Ausdehnung bis zur Linie Bitterfeld, Bad Düben, Torgau. Nach Osten erreichte das Programm eine Linie, die von Oschatz über Döbeln nach Mittweida ging. Nach Süden war die „Messewelle“ bis zur Linie Glauchau, Gera zu erreichen und nach Westen markierten Jena und Saalfeld das Ende des Empfangsgebietes. Mit dieser Verbreitung wurde die Zielfunktion Ausbreitungsradius $30 \mathrm{~km}$ erfüllt. ${ }^{104}$ Die Redaktion kam aufgrund der Hörerzuschriften und Meldungen der Funkamateure zu dem Resultat, dass die „Messewelle“ einen Ausbreitungsradius von etwa 70 Kilometern hatte. ${ }^{105}$

Da die Messe der Zeitpunkt war, um sich international auch tontechnisch vorzustellen, wurde während dieser Zeit von 12.05 bis 13.00 und 14.00 bis $15.00 \mathrm{Uhr}$ über die Frequenz 93,85 MHz von UKW-Leipzig [...] ein Stereo-Versuchsprogramm ausgestrablt. Dies sollte nach der Messe dienstags und donnerstags von 17.30 bis 18.00 Uhr weitergeführt werden. ${ }^{106}$ Hintergrund dieser Stereo-Versuchssendungen war die zentrale tonqualitative Neuerung im Hörfunk der 1960erJahre: die Einführung der Stereotechnik. Die DDR übertrug ihre ersten StereoSendungen aus Berlin und Leipzig, also den beiden größten Ballungsgebieten des Landes, aber vor allem den Orten mit der größten internationalen Öffentlichkeit. Mit den Stereo-Sendungen wurde der technische Gleichklang der DDR mit dem Ausland international akustisch aufgezeigt. Dass die Bevölkerung Mitte der 1960er-Jahre diese Stereoprogramme nicht empfangen konnte, da die ostdeutsche Industrie keine stereotauglichen Radiogeräte produzierte, unterstreicht die propa-

102 DRA, KV 5/71, 5. Februar 1971, Frühjahrsmesse 1971, Anlage Messewelle 22. Januar 1971.
103 DRA, KV 35/71, 20. Juli 1971, Herbstmesse 1971.
104 MDR, o. D., Konzeption Messewelle, Bl. 2.
105 Ebd.; TiETz, Erinnerungen (wie Anm. 5).
106 Funkecho 2/1966, 10. März 1966, o. p. 
gandistische Bedeutung der Stereo-Hörfunkprogramme. ${ }^{107}$ Vor allem die Versorgung mit Autoradios und portablen UKW-Empfängern war ein Problem.108 An den Frequenzen wurden nur minimale Veränderungen vorgenommen. Mit Ausnahme der Leipziger UKW-Frequenz, die teilweise variierte, war die „Messewelle" in den 1980er-Jahren über folgende Frequenzen zu empfangen:109

MW Leipzig $729 \mathrm{kHz}$, UKW Leipzig 98,5 MHz (von 7.00 bis $20.00 \mathrm{Uhr}$ )

UKW Leipzig 93,9 MHz (von 7.00 bis $10.00 \mathrm{Uhr}$ )

UKW Brocken 94,6, Dequede 94,9 MHz (von 8.00 bis $10.00 \mathrm{Uhr}$ )

Die Sendezeit der „Messewelle“ war Mitte der 1980er-Jahre von 7.00 bis 20.00 Uhr. ${ }^{110}$

\section{Hörerreaktionen - wer hört die „Messewelle“ wo?}

Obwohl die Messegäste als die erste Zielgruppe der „Messewelle“ genannt wurden, war ihre Meinung zum Programm stets sekundär. Ihre Einbeziehung ins Programm erfolgte in den Gesprächen mit ausländischen Ausstellern und Gästen und im Preisausschreiben. ${ }^{111}$ Man hörte sie beispielsweise in der Sendung „Was hat Ihnen die Leipziger Messe gegeben?", die als Radio DDR-Umfrage unter ausl[ändischen] Kauflenten mit Musik aus den Ländern der Gesprächspartner zwischen 19.30 und 21.00 Uhr auf die Gäste einging. ${ }^{112}$ Nur diejenigen Rückmeldungen, die man auf diese Art erhielt, waren als Publikumsreaktion der Gäste vorhanden. Eine organisierte oder gar qualitative Untersuchung der Hörermeinungen der Gäste erfolgte nicht.

Bedeutender als die Meinung der Gäste waren dagegen von jeher die Rückmeldungen der Leipziger. So diente als quantitativer Nachweis des Erfolgs und als Gelegenheit zur Ermittlung von Publikumsmeinungen vor allem das traditionelle Preisausschreiben von UKW Leipzig, an dem in erster Linie die Bevölkerung teilnahm. Zur Herbstmesse 1967 erhielt der Hörfunk damit interessante Angaben darüber, wann und wo unser Programm gehört wurde und daneben auch über die Reaktionen des Publikums. Die Frage nach dem wann und wo des Empfangs konnte mit den Einsendungen beantwortet werden, doch Antworten auf andere Fragen der Rezeption, gerade bei den Gästen, waren mit den Einsendungen zum Preisausschreiben schwerlich zu bekommen. Dennoch dienten diese Rückmel-

107 CHRISTIAN KÖNNE, Stereoradio für Berlin. Hörfunkmodernisierung im Wettlauf der Systeme, in: Michael Lemke (Hg.), Konfrontation und Wettbewerb. Wissenschaft, Technik und Kultur im geteilten Berliner Alltag 1948-1972, Berlin 2008, S. 135-160, hier S. 158.

108 Tietz, Erinnerungen (wie Anm. 5).

109 FF dabei 36/1986, S. 11 f.

110 FF dabei 36/1984, S. 37.

111 KÖHLER, Erinnerungen (wie Anm. 32).

112 DRA, KV 119/64, 6. Dezember 1964, Frühjahrsmesse 1965. 
dungen offenbar zusammen mit den bei jedem Sender eingehenden spontanen Äußerungen einzelner Hörer als Gradmesser für die eigene Arbeit und als Möglichkeit die weitere Programmentwicklung zu planen.

Die Rückmeldung durch die ostdeutsche Bevölkerung war noch in weiterer Hinsicht für das Programm aus Leipzig relevant. Offenbar wollte man das internationale Ereignis Leipziger Messe landesweit besser in der Wahrnehmung der Bevölkerung verankern - auch hierzu diente das Preisausschreiben. Die Ergebnisse des Jahres 1967 waren daher insofern wichtig, als hier erstmalig auch Beiträge des Preisausschreibens am Nachmittag über das Mittelwellen-und UKW-Sendenetz des I. Programms von Radio DDR liefen, die in der ganzen Republik empfangen werden konnten. Der Versuch, die Messesendungen landesweit zu etablieren, scheiterte: Trotz des bei weitem größeren Einzugsbereiches für diese Sendungen [...] ist die Hörbeteiligung hier nicht wesentlich größer als zu den [...] Frühsendezeiten im Regionalprogramm. ${ }^{113}$ Das Angebot war also nur regional bedeutsam. Die übrige DDR war selbst mit einem Preisausschreiben nicht für das Sonderprogramm aus Leipzig zu gewinnen, wenngleich man einschränkend hinzufügen muss, dass die Sendezeiten für die landesweiten Übertragungen nicht in die Hauptnutzungszeit des Mediums fielen. Anteilig an den Zuschriften und Anrufen aus den Bezirken auf die Sendungen im Regionalprogramm ergab dies 1967 folgendes Bild:114

\begin{tabular}{|l|c|l|c|}
\hline \multicolumn{4}{|c|}{ Höreranteil des Leipziger Sonderprogramms } \\
\hline Zeit & $6.20 \mathrm{Uhr}$ & $8.30 \mathrm{Uhr}$ & $13.10 \mathrm{Uhr}$ \\
\hline Bezirk Leipzig & $49,7 \%$ & $47,6 \%$ & $67,2 \%$ \\
\hline Bezirk Halle & $14,6 \%$ & $14,4 \%$ & $11,6 \%$ \\
\hline Bezirk Magdeburg & $7,6 \%$ & $12,2 \%$ & $1,6 \%$ \\
\hline übrige DDR & $28,1 \%$ & $25,8 \%$ & $19,6 \%$ \\
\hline
\end{tabular}

Die „Messewelle“ war nach diesen Werten überwiegend regional relevant, das stand Ende der 1960er-Jahre fest. Die Bezirke Leipzig, Halle und Magdeburg gehörten von der Seite des Hörfunks her gesehen enger zusammen. Bis zur Etablierung von eigenen Regionalsendern in diesen Bezirken, hatte der Sender Leipzig für diese Bezirke eine Mitversorgungspflicht, weshalb die Untersuchung diese separat ausweist. Der Bezirk Dresden wurde nicht genauer abgebildet, obwohl das ebenfalls sächsisches Sendegebiet war. Dresden hatte jedoch innerhalb von Radio DDR einen eigenen Regionalsender.

Die schriftlichen und telefonischen Reaktionen des Publikums auf das Leipziger Programm wurden rein zahlenmäßig festgehalten. Eine qualitative Auswertung der Publikumsreaktionen findet sich nicht. Im ersten Jahr der „Messewelle“, 1971, wurde die Hörerresonanz für die Frühjahrsmesse auf 1.155 und für die Herbstmesse auf 1.285 Hörerkontakte beziffert. Fünf Jahre später, 1976, erhielt

113 Funkecho 2/1967, 20. April 1967, S. 43.

114 Ebd. 
die "Messewelle“ während der Frühjahrsmesse 8.271 (+ $616 \%)$ und für die Herbstmesse 11.856 (+ $822 \%$ ) Meldungen vom Publikum. Nach weiteren fünf Jahren hatten sich diese zur Frühjahrsmesse 1981 auf 13.000 (+ 57,1\%) und zur Herbstmesse auf 8.500 (-28,4 \%) verändert. Die Frühjahrsmesse erhielt 1989 dann 46.057 und die Messe des Wendeherbstes 1989 schließlich 34.998 Reaktionen. Im Frühjahr 1990 beteiligten sich noch 11.997 Hörer an der "Messewelle“.115 Die einzige differenzierte Darstellung der Hörerreaktionen liegt für die Herbstmessen der Jahre 1989 und 1990 vor:116

\begin{tabular}{|c|c|c|c|c|c|c|c|c|c|}
\hline & \multicolumn{4}{|c|}{ Herbstmesse 1989} & \multicolumn{4}{|c|}{ Herbstmesse 1990} & \multirow{2}{*}{$\begin{array}{c}\text { Änderung } \\
1989-90\end{array}$} \\
\hline & $\begin{array}{l}\text { telefo- } \\
\text { nisch }\end{array}$ & $\begin{array}{l}\text { schrift- } \\
\text { lich }\end{array}$ & $\begin{array}{c}\text { gesamt } \\
1989 \\
\end{array}$ & $\begin{array}{c}\text { Anteil } \\
\%\end{array}$ & $\begin{array}{c}\text { telefo- } \\
\text { nisch }\end{array}$ & $\begin{array}{c}\text { schrift- } \\
\text { lich }\end{array}$ & $\begin{array}{c}\text { gesamt } \\
1990\end{array}$ & $\begin{array}{c}\text { Anteil } \\
\%\end{array}$ & \\
\hline $\begin{array}{l}\text { Preisaus- } \\
\text { schreiben }\end{array}$ & 16.655 & 11.945 & 28.598 & 81,7 & 5.535 & 2.691 & 8.226 & 68,5 & $-71,3 \%$ \\
\hline Grüße & 1.065 & 894 & 1.959 & 5,5 & 477 & 220 & 697 & 5,8 & $-64,5 \%$ \\
\hline $\begin{array}{l}\text { Sächsisch } \\
\text { im Funk }\end{array}$ & 1.534 & 579 & 2.113 & 6,0 & 1.737 & 290 & 2.027 & 16,8 & $-4,1 \%$ \\
\hline Hits & 444 & 1.351 & 2.328 & 6,6 & 265 & 735 & 1.000 & 8,3 & $-57,1 \%$ \\
\hline Sonstiges & 0 & 0 & 0 & 0 & 31 & 11 & 42 & 0,3 & $+0,3 \%$ \\
\hline Gesamt & 19.698 & 14.769 & 34.998 & 100 & 8.045 & 3.947 & 11.997 & 100 & $-65,8 \%$ \\
\hline
\end{tabular}

Die Auswertung der „Messewelle“ zeigt, dass die Menschen das Sonderprogramm unverändert vor allem für das Preisausschreiben, gefolgt von den Zuschriften für die „Hitparade“ und für die Reihe „S̈̈chsisch im Funk“ nutzten. Nur 42 Rückmeldungen wurden unter der Rubrik „Sonstiges“ gezählt. Die Ausrichtung auf die sprachlich in sächsischer Manier gestalteten Programmbereiche hatte offenbar entsprechenden Widerhall beim Publikum gefunden. In einer Mangelgesellschaft Preise gewinnen zu können, war offenbar ein großes Stimulans für eine Teilnahme. Doch noch etwas anderes ist bemerkenswert. Die Hörerreaktionen zu „Sächsisch im Funk" und zur "Sächsischen Hitparade“ sind die stabilsten. Hatte die Idee der lokalen Identifikation funktioniert?

Noch eine andere Tatsache fällt auf. Obwohl die „Messewelle“ als ein ServiceProgramm für die internationalen Gäste der Leipziger Messe angekündigt wurde und auch so geplant war, fragte man diese nie gezielt nach deren Meinung zum Programm. Alle Informationen, die die „Messewelle“ zum Programm von den Messegästen erhielt, erfuhren die Mitarbeiter quasi nebenbei. Nach Angaben eines ehemaligen Redakteurs haben ausländische Gäste das Programm als hörenswert und nützlich bezeichnet. Die Meinung des ureigenen Publikums, der Messegäste, war zu unwichtig oder politisch zu brisant, um abgefragt zu werden. Woher das angebliche Wissen über die Wirksamkeit und den vermeintlichen Erfolg des „Messe-Service“ kam, ist also die Frage. Sicher kam es nicht von der Zielgruppe. Die einzigen erhaltenen Aussagen von Nicht-DDR-Einwohnern sind diesem

115 MDR, o. D., Hörerresonanz Messewelle, Bl. 38.

116 MDR, 8. September 1990, Hörerbeteiligung Messewelle, Bl. 37. 
Fokus in der Bedeutung der ausländischen Gäste gemäß nicht solche, die als diejenigen von Messebesuchern in Form von nützlichen Informationen ausgewertet wurden. Die Zusendungen liefen als Post von, weiter her'. Lediglich drei dieser Äußerungen sind erhalten, die keine inhaltlich relevanten Informationen enthalten. Die Messegäste scheinen für den Hörfunk der DDR in puncto „Messewelle“ uninteressant gewesen zu sein. ${ }^{117}$

Dass das Programm bei der eigenen Bevölkerung ein Erfolgskonzept für den Regionalsender Leipzig war, wurde sehr wohl untersucht. Seit den 1960er-Jahren waren es die Zusendungen aus der Region Leipzig, die als Gradmesser dienten. Letztmalig bestätigte dies eine Untersuchung der Hörgewohnheiten in den Jahren 1987/88. Auch hier wurde allein die Bevölkerung des Bezirks, nicht aber die Messebesucher befragt. Bei der Bezirksbevölkerung über 15 Jahren lag die tägliche Hörerquote des Regionalsenders Leipzig im Wochentagesdurchschnitt von Montag bis Sonntag während der Zeit der „Messewelle“ bei $28 \%$, außerhalb der Zeit dagegen nur bei $13 \% .{ }^{118}$ Das Sonderprogramm war offenbar geeignet, die Hörerquote für das Regionalprogramm deutlich zu erhöhen.

\section{Fazit}

Zwei Vorläufer waren es, deren Erfahrungen und Ziele in die 1971 etablierte „Messewelle“ einflossen - der „Werbefunk“ und der „Service“ für die Gäste. Daneben fand im "Service“ von Beginn an auch eine sächsische Mundartunterhaltung ihren Platz.

Hörfunk anlässlich der Leipziger Messe hatte seit jeher eine wirtschaftliche und eine politische Komponente. Im Zusammenhang der finanziellen Schwierigkeiten der DDR zu Beginn der 1960er-Jahre wurde Werbung aus dem westlichen Ausland gezielt zur Finanzierung des Sozialismus im Programm platziert. Auch in anderer Weise ging man stärker auf den Westen zu, als dies bis dato denkbar gewesen war. Die Handelspartner wurden mit separatem Etat auch vom ostdeutschen Hörfunk auf der Messe in Leipzig umworben. Das war eine Entwicklung, die im Zusammenhang mit dem „Neuen Ökonomischen System der Planung und Leitung" stand und in der Folge des VI. Parteitags möglich wurde. Es war ein Modell, von dem sich die SED und der Hörfunk der DDR konkrete finanzielle Erleichterung versprachen. Gleichzeitig konnte durch die größere wirtschaftliche Kooperation mit westeuropäischen Handelspartnern vielleicht die Abgrenzung gegenüber der Bundesrepublik im Rahmen der sogenannten Störfreimachung besser vollzogen, in jedem Falle aber deutlich im Programm abgebildet werden. Die DDR erbrachte damit, vor ihrer internationalen Anerkennung, den Nachweis

117 MDR, o. D., Post von „weiter her“, Bl. 10.

118 DRA, Abteilung Soziologische Forschung, 017-01-02, Juni 1989: Uwe MeERGans, Rundfunk in der Region. Forschungsbericht zu empirischen Untersuchungen 1985-89. 
ihrer internationalen Hoffähigkeit gerade auch bei westeuropäischen (Handels-) Partnern. Dies war es wert, landesweit im Programm von Radio DDR I übertragen zu werden.

Doch die Zeit der Messe war auch in anderer Hinsicht Zeit für ein außergewöhnliches Hörfunkprogramm. Radio aus Leipzig war Mitte der 1960er-Jahre von der Sendezeit sowie technisch und inhaltlich das modernste ostdeutsche Programmangebot. Hier hatte DDR-Radio das stets erstrebte Weltniveau - die ausländischen Gäste aus aller Welt sollten das hören und kolportieren.

Gestartet waren die Hörfunksendungen des Regionalsenders anlässlich der Leipziger Messe als Service für ausländische Gäste und Leipziger Gastgeber - zwei Zielgruppen wurden bedient. In dieser Hinsicht arbeitete der Sender Leipzig konzeptionell ähnlich, wie dies beispielsweise der Sender Rostock mit der „Ferienwelle“ tat. In Leipzig war die ausländische (Hörer-)Gruppe aber die gewichtigere. Denn der „Messe-Service“ war originär für die Gäste aufgenommen worden. Durch den Erfolg des Service, der bemerkenswerter Weise mit dem Preisausschreiben (bei der Leipziger Bezirksbevölkerung?) gemessen wurde, fand eine allmähliche Ausdifferenzierung des Programmangebots statt. Hierbei ist für den Service ein Fokus auf die Interessen und Bedürfnisse der Gäste zu erkennen. Mit lediglich einer unterhaltenden 3-Minuten-Reihe auf Sächsisch hatten die Belange der Gäste bis 1971 Vorrang vor der Unterhaltung für die Leipziger. Doch änderte sich diese Ausrichtung des Programms in Bezug auf die Haupthörgruppen.

Zum Start der „Messewelle“ 1971 waren beide Komponenten, also die Informationen für die Messebesucher, daneben aber auch verstärkt Sendereihen, die sich unterhaltend vor allem an die Bevölkerung des Bezirks wandten, vorhanden. Bemerkenswert ist in Bezug auf die ausländischen Gäste die Tatsache, dass mit dem Beginn der „Messewelle“ die Werbesendungen auf das Sonderprogramm aus Leipzig reduziert wurden - denn dies brachte den beworbenen Produkten weniger Öffentlichkeit. Doch war die internationale Anerkennung der DDR zu dieser Zeit vollzogen, sodass aus diesem Grunde eine landesweite Übertragung von West-Werbung nicht mehr nötig war. Nicht zuletzt stellte sich bei solcher Werbung auch die Frage, welche Produkte beworben wurden und inwieweit sich die Bevölkerung der DDR diese Produkte überhaupt leisten konnte. Schließlich bildete auch die internationale musikalische Ausgestaltung des Programms die Situation in der Stadt musikalisch im Programm ab. Die „Radio-DDR-Messewelle“ bot modernen Hörfunk und musikalisch internationales Flair - zweimal jährlich für die Gäste - aber eben auch für die Bezirksbevölkerung.

Mitte der 1970er-Jahre kam es zu Veränderungen, die Innovationen der „Messewelle“ mit sich brachten. Das sächsische und das städtische Element Leipzigs wurden prägend für das Programm. Eine Verschiebung in der Gewichtung beider Zielgruppen fand statt. Nunmehr lagen beide Zielgruppen, also die Bezirksbevölkerung im Unterhaltungsteil und weiterhin die Gäste im Serviceteil im Interesse der Programmgestalter. Dass die SED dies förderte, lag an den politischen Zielen, 
die sie mit den Regionalprogrammen verfolgte. Die regionale Bindung der Menschen sollte erreicht werden und bei diesen schließlich ein DDR-Heimatbewusstsein evozieren, das zur Abgrenzung gegenüber der Bundesrepublik diente. Mit dieser Umgestaltung war die „Messewelle“ seit Mitte der 1970er-Jahre ein unterhaltendes Programm mit speziellem Dialekt-Herkommen auf Sächsisch für die Bevölkerung der Region Leipzig, das unverändert auch die Informationen bot, die die ausländischen Messegäste brauchten. Vom Sendeanteil und von der Sprache her hatte man sich damit von den Gästen und auch der übrigen DDR abgekoppelt. Die Absonderung des Bezirks hielt bis zu den 1980er-Jahren an. Die „Messewelle“ aus Leipzig war nun vor allem ein regionales Highlight für die Bevölkerung der Stadt - das Schmankerl zur Messe.

Die wirtschaftlichen Zwänge der 1980er-Jahre brachten jedoch auch eine erneute Fokussierung der ausländischen Partner in der „Messewelle“ mit sich. Wie schon zuvor wurde die vom Westen finanzierte Werbung zu einem zentralen Element der „Messewelle“ - damit hörte man im Programm aus Leipzig in der Tat Töne, die für DDR-Verhältnisse außergewöhnlich waren. Akustisch war die Werbung international - wirtschaftlich notwendig. Dass die Wiederaufnahme der Werbung offenbar sehr plötzlich, aber dafür mit relativ hohem Aufwand an Personal betrieben wurde, unterstreicht die Bedeutsamkeit, die der Hörfunk dem Werbefunk zuwies.

Die Publikumsreaktionen kamen vor allem zu den unterhaltenden Teilen des Programms. Das Konzept durch Unterhaltung einen Kontakt der Menschen zum System zu erreichen, funktionierte offenbar. So erhielt man die konstantesten Zuschriften zu „Sächsisch im Funk“. Ob dies zur gewünschten Identifikation führte, wurde nicht untersucht. Es ist jedoch die Frage, ob das für die Wahrnehmung des Erfolgs durch die SED später noch relevant war. Für die Partei genügte die Teilnahme der Menschen an den offiziellen Programmen als Nachweis des politisch erstrebten Erfolgs.

An den Publikumsreaktionen ist ebenfalls erstaunlich, dass die Interessen der Menschen aus der Region Leipzig zur weiteren Programmentwicklung sehr wohl zur Kenntnis genommen wurden. Umgekehrt wurden jedoch die Interessen der internationalen Gäste, für die man das Programm ja eigentlich initiiert hatte, nie erfragt. Ob und wie diese mit ,ihrem' Programm zufrieden waren, wurde nicht untersucht. Auch hieraus ergibt sich die Relevanz in der Bewertung der „Messewelle" in Bezug auf die Funktionen eines regionalen Programmangebots für die Identitätsbildung durch die Programmverantwortlichen.

Doch unabhängig davon war die „Messewelle“ in jedem Falle ein mediales Sonderangebot, das der Leipziger Messe eine spezielle Note hinzufügte - und wer genau diese dann hörte, war egal. Man würde von diesem Programm erzählen. 